\title{
Role of dimorphism in the development of Candida albicans biofilms
}

\author{
GEORGE S. BAILLIE and L. JULIA DOUGLAS \\ Division of Infection and Immunity, Institute of Biomedical and Life Sciences, University of Glasgow, Glasgow, \\ G12 800
}

Two model biofilm systems, involving growth on disks of catheter material or on cylindrical cellulose filters, were used to investigate the structure of Candida albicans biofilms. To assess the importance of dimorphism in biofilm development, biofilms produced by two wild-type strains were compared with those formed by two morphological mutants, incapable of yeast and hyphal growth, respectively. Scanning electron microscopy and thin sections of biofilms examined by light microscopy revealed that biofilms of the wild-type strains formed on catheter disks consisted of two distinct layers: a thin, basal yeast layer and a thicker, but more open, hyphal layer. The hypha ${ }^{-}$ mutant produced only the basal layer, whereas the yeast ${ }^{-}$mutant formed a thicker, hyphal biofilm equivalent to the outer zone of the wild-type structures. Biofilms of the yeast $^{-}$mutant were more easily detached from the catheter surface than the others, suggesting that the basal yeast layer has an important role in anchoring the biofilm to the surface. Biofilms formed on cylindrical cellulose filters were quite different in appearance. The hypha ${ }^{-}$mutant and both wild types produced exclusively yeast-form biofilms whereas the yeast ${ }^{-}$mutant generated a dense hyphal mat on the top of the filter. All these biofilms, irrespective of morphological form, were resistant to the antifungal agent, amphotericin B. Overall, these results indicate that the structure of a $C$. albicans biofilm depends on the nature of the contact surface, but that some surfaces produce biofilms with a layered architecture resembling to that described for bacterial systems.

\section{Introduction}

Medical implants, such as catheters, prosthetic heart valves and joint replacements, are all susceptible to colonisation by micro-organisms which can attach to the device and form a biofilm on its surface. Biofilms consist of layers of microbial cells embedded within a matrix of extracellular polymeric material [1-3]. Release of micro-organisms from the biofilm can result in an acute disseminated infection which may be responsive to antimicrobial therapy. However, the biofilm itself is generally resistant, and will frequently persist as a reservoir of infection until the implant is removed $[4,5]$. Although the majority of implant infections are caused by bacteria - notably staphylococci - fungal infections are becoming increasingly

Received 18 Aug. 1998; accepted 6 Nov. 1998.

Corresponding author: Dr L. J. Douglas (e-mail:

J.Douglas@bio.gla.ac.uk). common and Candida species are regarded as particularly important nosocomial pathogens [6].

Bacterial biofilms have been investigated in detail over a number of years and there is now a considerable amount of information available relating to their structure and properties [2, 7-9]. Much less is known about fungal biofilms and relatively few model systems have been used for their study in vitro. A simple system for Candida biofilms involves growing adherent populations on the surfaces of small disks of catheter material [10-12]. Growth can be monitored quantitatively by dry weight measurements and by colorimetric or radio-isotope assays [10]. Biofilms of C. albicans produced in this way consisted of a dense network of yeasts, hyphae and pseudohyphae, together with a matrix material whose synthesis increased markedly when developing biofilms were subjected to a liquid flow [12]. The biofilms were resistant to a variety of antifungal agents, including amphotericin B and fluconazole [11]. 
To produce controlled growth of Candida biofilms, a second system, the perfused biofilm fermenter, has been used. Here, a biofilm is formed on the underside of a cellulose acetate membrane which is perfused with growth medium. Reproducible growth-rate control can be achieved and this system has been used to investigate the effect of growth rate on the resistance of Candida biofilms to antifungal agents [13]. In a third model system, biofilms were formed within cylindrical cellulose filters perfused with medium. This apparatus lacks stainless steel components and was used to produce iron-limited Candida biofilms at low growth rates [14].

The present study used two of these model systems, the catheter material disk and cylindrical cellulose filter, to investigate the detailed structure of $C$. albicans biofilms and, in particular, the possible role of dimorphism in biofilm development. Biofilms produced by two wild-type strains of $C$. albicans were compared with those formed by two morphological mutants, incapable of yeast and hypha formation, respectively.

\section{Materials and methods}

\section{Organisms and growth media}

Four strains of $C$. albicans were used in this study. $C$. albicans GDH 2346 (NCYC 1467) was isolated at Glasgow Dental Hospital from a patient with denture stomatitis and $C$. albicans 1001 (ATCC 64385) was obtained originally from a case of systemic candidosis. Two mutants $[15,16]$ derived from strain 1001 and unable to form yeasts (strain 1001-FR; yeast ${ }^{-}$) or hyphae (strain 1001-92'; hypha ${ }^{-}$) were supplied by $\mathrm{C}$. Gil, Universidad Complutense, Madrid, Spain. All organisms were maintained on slopes of Sabouraud Dextrose Agar (Difco) and subcultured monthly. Every 2 months, cultures were replaced by new cultures freshly grown from freeze-dried stocks. For biofilm formation, organisms were grown in Yeast Nitrogen Base Medium (Difco) containing $50 \mathrm{mM}$ glucose (catheter disk procedure) or $4 \mathrm{mM}$ glucose (cylindrical filter procedure).

\section{Biofilm formation on catheter disks}

Biofilms were grown on small disks (surface area, $0.5 \mathrm{~cm}^{2}$ ) cut from polyvinyl chloride (PVC) Faucher tubes (French gauge 36; Vygon, Cirencester), as described previously [10]. Briefly, the disks were placed in wells of 24-well Nunclon tissue culture plates and a standardised cell suspension $(80 \mu \mathrm{l})$ was applied to the surface of each one. After incubation for $1 \mathrm{~h}$ at $37^{\circ} \mathrm{C}$ (adhesion period), non-adherent organisms were removed by washing with $0.15 \mathrm{M}$ phosphatebuffered saline (pH 7.2; PBS). The disks were then incubated in the wells for $24 \mathrm{~h}$ at $37^{\circ} \mathrm{C}$, submerged in $1 \mathrm{ml}$ of growth medium (biofilm formation).

\section{Biofilm formation on cylindrical cellulose filters}

Biofilms were grown on cylindrical filters consisting of compacted cellulose fibres (Gilson safety filters; $22 \times 8 \mathrm{~mm}$; Anachem, Luton) as described previously [14]. Each filter was inserted into silicone tubing attached to the bottom of a disposable syringe body $(2 \mathrm{ml})$, from which the plunger had been removed. Medium was pumped directly into the vertically clamped syringe body via silicone tubing. Filters were pre-wetted with sterile saline $(5 \mathrm{ml})$ and then inoculated with an exponential-phase batch culture $(10 \mathrm{ml})$ whose optical density at $540 \mathrm{~nm}$ had been adjusted to a value of 0.8 . After perfusion of the inoculum, the filters were perfused with medium at a flow rate of $0.87 \mathrm{ml} / \mathrm{min}$. The eluate passing through the filter was collected at various time intervals and viable counts were made by serial dilution in $0.15 \mathrm{MPBS}$ and plating in triplicate on Sabouraud dextrose agar. The plates were incubated at $37^{\circ} \mathrm{C}$ for $16 \mathrm{~h}$ before counting. This gave an estimate of the numbers of newly formed daughter cells. Growth rates of biofilms (divisions/h) were calculated by dividing the number of daughter cells produced per hour at steady state by the estimated adherent cell population (determined by viable counts of resuspended biofilms). All biofilms were grown for at least $24 \mathrm{~h}$ under steady state conditions before treatment with amphotericin B.

\section{Measurement of strength of biofilm attachment to catheter disks}

A plate succession technique [17], designed to quantify the ease of removal of bacteria from surfaces, was modified to determine the strength of attachment of biofilm cells to PVC catheter disks. Biofilms were grown for $24 \mathrm{~h}$ on disks as described above. The disks were then removed from the culture medium and plunged sequentially (biofilm face down) into a series of wells containing $1 \mathrm{ml}$ of saline. The number of cells lost from each biofilm as it was forced through each air/liquid interface was determined by serial dilution and plating in duplicate on Sabouraud dextrose agar. Cell loss per interface was plotted against interface number and the removal exponent $(\mathrm{k})$ was taken as the slope of the resulting straight line.

\section{Susceptibility of biofilm cells to amphotericin B}

The susceptibility to amphotericin B of steady-state biofilm cells (grown on cylindrical filters), resuspended biofilm cells and newly formed daughter cells was determined by a method described previously [14]. An amphotericin B concentration of $0.1 \mathrm{mg} / \mathrm{L}$ was used as this concentration gave a reduction in viability of $>80 \%$ when planktonic cells of $C$. albicans were tested by the same procedure [13]. Briefly, cylindrical cellulose filters with adherent biofilms were cut in half longitudinally. One half of each filter was immersed in amphotericin $\mathrm{B}$ solution for $1 \mathrm{~h}$ at $37^{\circ} \mathrm{C}$; the adherent cells were then resuspended by breaking up the filter 
with a sterile aluminium rod followed by vigorous vortex mixing for $1 \mathrm{~min}$. Cells on the other half of the filter were first resuspended in sterile water in an identical fashion, and samples of the suspension were added to amphotericin $\mathrm{B}$ solution and incubated at $37^{\circ} \mathrm{C}$ for $1 \mathrm{~h}$. Samples of the eluate from the filter which contained daughter cells were also added to a solution of the drug and incubated similarly for $1 \mathrm{~h}$. Viable counts were made on all samples by serial dilution and plating in triplicate on Sabouraud dextrose agar. Figures for percentage survival were calculated by comparison with counts for untreated control samples processed similarly.

\section{Microscopy}

Biofilms formed on PVC catheter disks or on cylindrical cellulose filters were fixed with glutaraldehyde, then treated with osmium tetroxide and uranyl acetate as described previously [10]. After dehydration in a series of ethanol solutions, samples were dried overnight in a desiccator, coated with gold with a Polaron coater and viewed with a Philips 500 scanning electron microscope.

Biofilm structure was also observed by light microscopy after biofilms had been sectioned. Samples were prepared for thin-sectioning as follows. Biofilms grown on catheter disks were fixed with glutaraldehyde $2.5 \% \mathrm{v} / \mathrm{v}$ in $0.15 \mathrm{M}$ PBS for $1 \mathrm{~h}$ at room temperature, washed three times with $0.15 \mathrm{M}$ PBS, and treated with osmium tetroxide $2 \% \mathrm{w} / \mathrm{v}$ for $1 \mathrm{~h}$. After two further washes in distilled water, biofilms were captured in agar $1.5 \%$. The agar blocks were then treated with uranyl acetate $0.5 \%$, dehydrated in a series of ethanol solutions, and left overnight in a 1:1 mixture of absolute ethanol and Spurr resin. After evaporation of ethanol from the samples, they were transferred to tubes containing fresh Spurr resin and rotated for $16 \mathrm{~h}$ before polymerisation of the resin at $60^{\circ} \mathrm{C}$ for $24 \mathrm{~h}$. Sections $(1 \mu \mathrm{m})$ were cut with an LKB Ultrotome III and stained in toluidine blue $1 \%$ containing borax $1 \%$ at $60^{\circ} \mathrm{C}$ for $10 \mathrm{~s}$.

\section{Results}

\section{Biofilm populations on PVC disks}

The formation of biofilms on small disks cut from PVC catheters was monitored by scanning electron microscopy (SEM). Biofilm development by the wild-type strain 1001 was similar to that previously reported for C. albicans GDH 2346, another clinical isolate [10]. Initial adhesion by cells exclusively in the yeast phase was followed by a period of budding and germ-tube formation. After $24 \mathrm{~h}$, a thick biofilm had formed containing hyphae, pseudohyphae and yeasts in a three dimensional mesh (Fig. 1). Thin sections of biofilms produced by these dimorphic strains (Fig. 2) revealed two distinct zones: a basal region which comprised
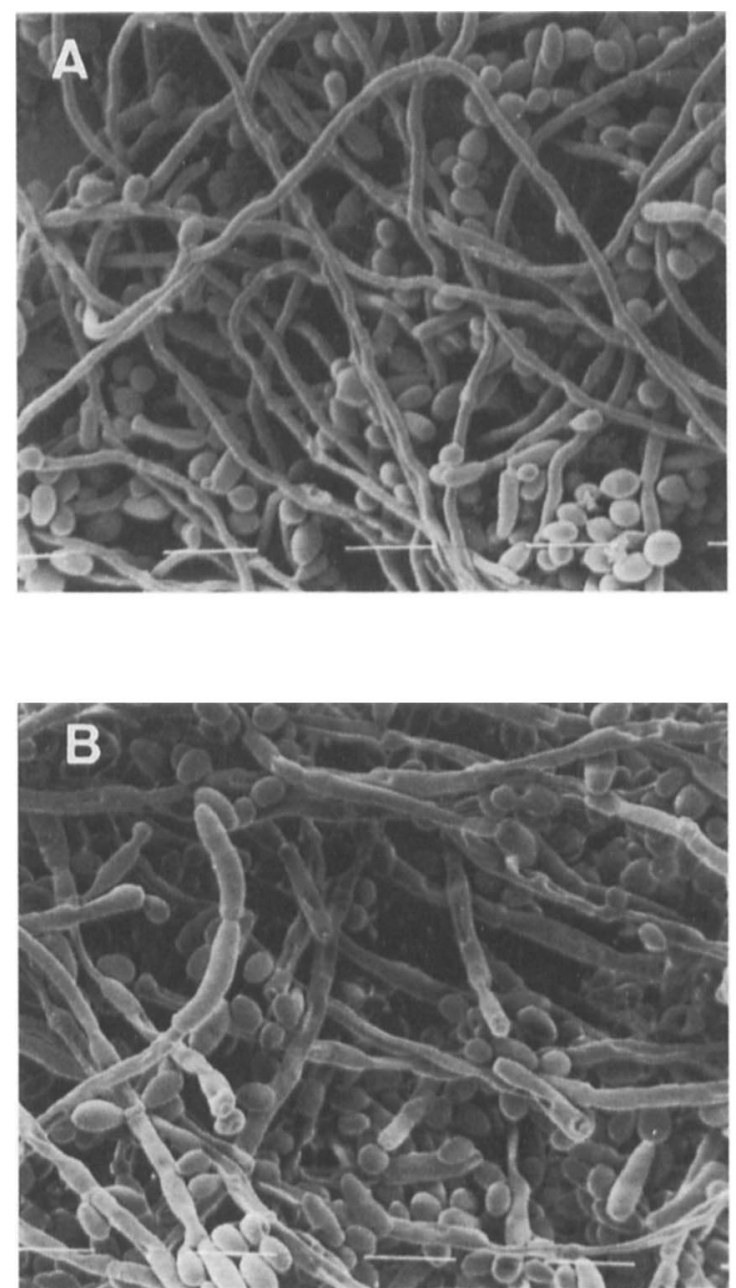

Fig. 1. SEM of biofilms formed by $C$. albicans GDH 2346 (A) and C. albicans 1001 (B) on PVC catheter disks after incubation for $24 \mathrm{~h}$. Bar, $10 \mu \mathrm{m}$.

tightly packed cells mostly in the yeast form (approximately one-quarter of the total biofilm depth) and a more open, outer zone consisting largely of hyphae (three-quarters of the depth). This bilayer structure could also be seen by SEM in regions where the hyphal mesh was pulled back or had broken off.

The morphological mutant incapable of hypha formation (strain 1001-92') adhered to PVC disks in a uniform manner and after $5 \mathrm{~h}$ there was partial coverage of the surface by budding yeast cells (Fig. $3 \mathrm{~A})$. By $24 \mathrm{~h}$, a dense yeast biofilm, devoid of hyphae or germ-tubes, had developed (Fig. 3B). The open architecture produced as a result of hypha formation was absent and the biofilm seemed less deep than that of the wild type. In contrast, biofilm formation by the mutant incapable of yeast growth (strain 1001-FR) consisted initially of adherent clumps of hyphae and pseudohyphae on the PVC surface. After $5 \mathrm{~h}$, adjacent clumps had become linked via germ-tube formation into a sparse network of hyphal biofilm (Fig. 4A). Further growth resulted in a more extensive hyphal 

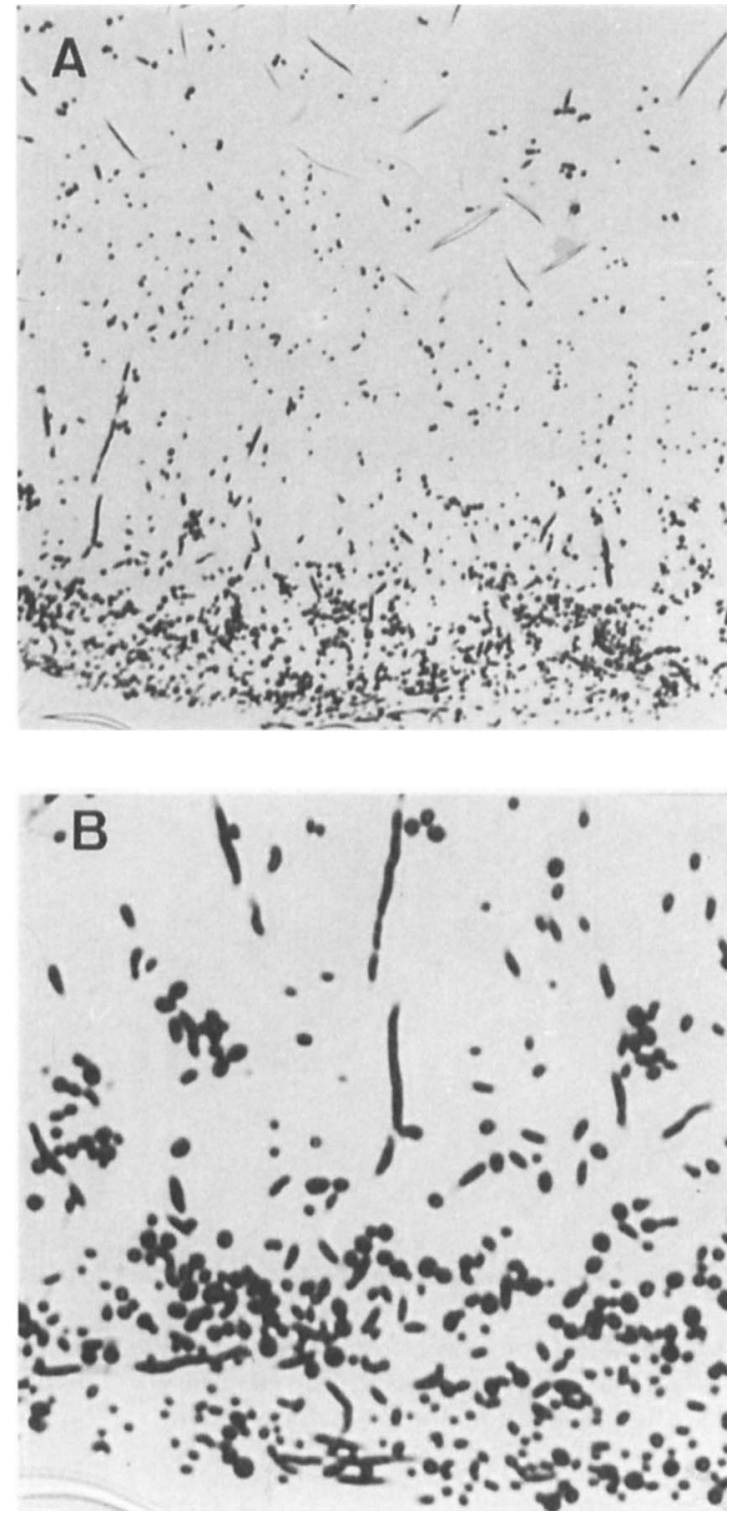

Fig. 2. Thin sections of a biofilm formed by C. albicans GDH 2346 on a PVC catheter disk and viewed by light microscopy. Final magnification, $465 \times(\mathrm{A})$ and $1163 \times(\mathbf{B})$.

network with hyphae extending outwards into the bulk fluid (Fig. 4B). Overall, hyphal biofilms appeared less dense than either those consisting of yeasts only or those containing both morphological forms.

\section{Strength of biofilm attachment to PVC disks}

The strength of attachment of biofilm organisms was determined by a modification of the plate succession technique [17] devised for bacterial biofilms. In the modified method, a catheter disk with its fully formed biofilm is plunged into a series of wells containing saline; the removal exponent $(\mathrm{k})$ provides a convenient and reproducible measure of the ease of removal of the organisms from the disk, higher values indicating a looser attachment. Experiments of this type were
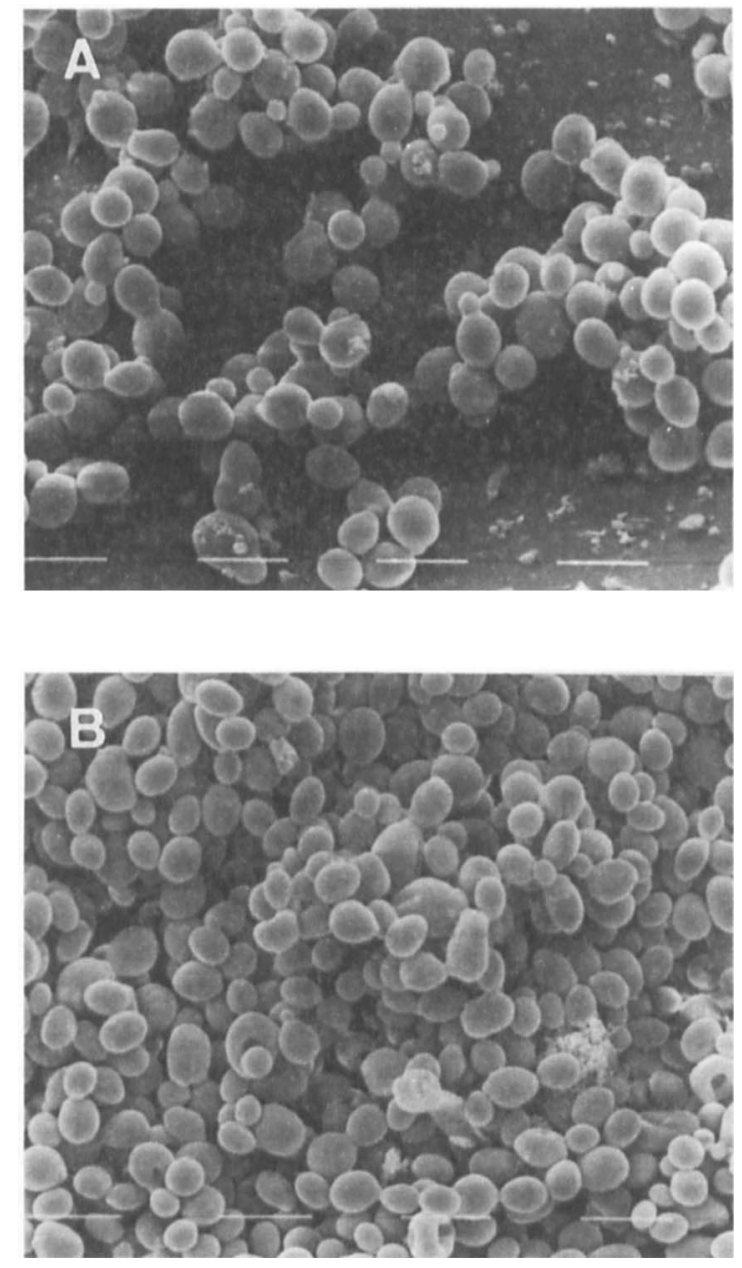

Fig. 3. SEM of biofilms formed by $C$. albicans 1001-92' on PVC catheter disks after incubation for $5 \mathrm{~h}(\mathbf{A})$ and $24 \mathrm{~h}$ (B). Bar, $10 \mu \mathrm{m}$.

performed with strains GDH 2346 and 1001, and both morphological mutants. The results (Fig. 5) clearly showed that biofilms containing only yeasts (strain 1001-92') and those containing a mixture of morphological forms (strains GDH 2346 and 1001) had similar removal exponents $(\mathrm{k}=0.21,0.21$ and 0.23 , respectively). However, hyphal biofilms produced by strain 1001-FR displayed a significantly higher removal exponent $(\mathrm{k}=0.40 ; \mathrm{p}<0.05)$, indicating that they were less well anchored to the PVC surface.

\section{Biofilm populations on cylindrical cellulose filters}

Biofilms of all four strains were also grown on cylindrical cellulose filters perfused with growth medium. The elution profiles of cells released from these biofilms (Fig. 6) showed that initial loss of free or loosely attached cells occurred within the first hour of culture. This was then followed by a period of biofilm growth during which adherent populations reached an optimal density. With both wild-type strains, daughter cells were eluted at a constant rate after $5 \mathrm{~h}$, indicating that a steady state had been attained. However, the 

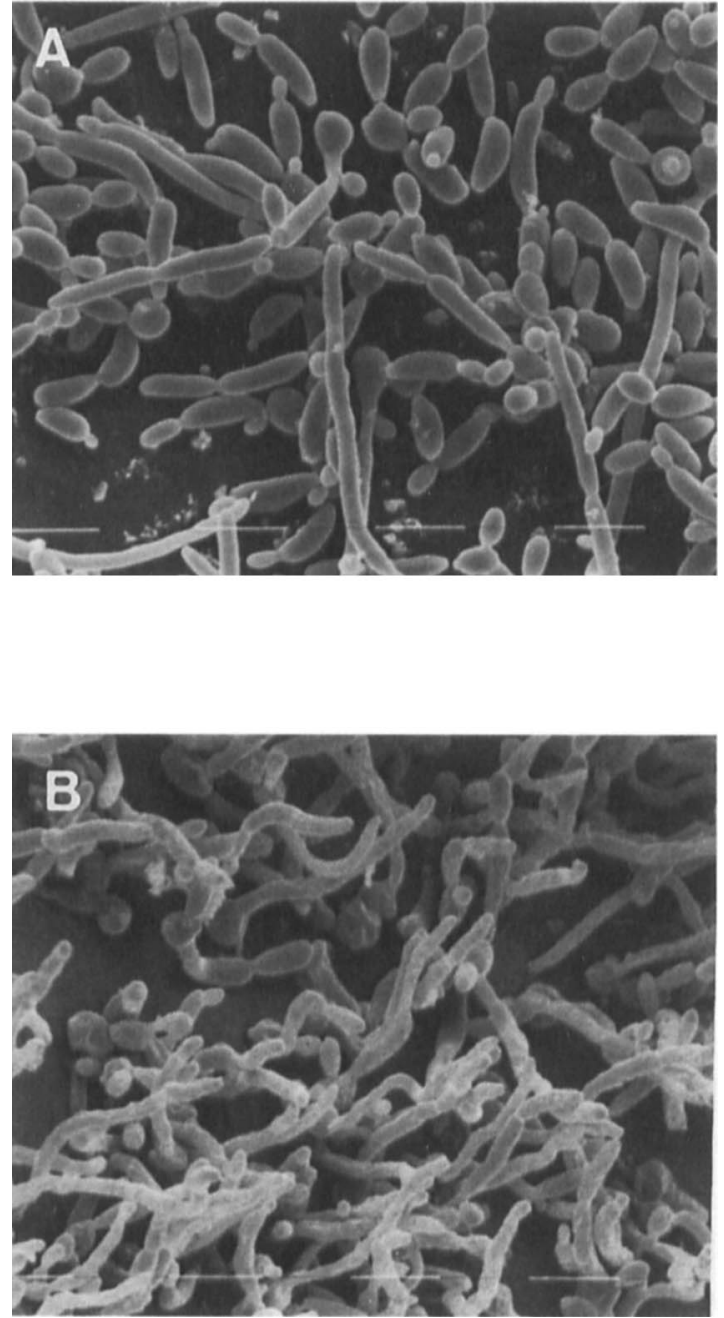

Fig. 4. SEM of biofilms formed by C. albicans 1001-FR on PVC catheter disks after incubation for $5 \mathrm{~h}$ (A) and $24 \mathrm{~h}$ (B). Bar, $10 \mu \mathrm{m}$.

morphological mutants took much longer $(24 \mathrm{~h})$ to reach this stage, and the numbers of daughter cells eluted at steady state were lower (Fig. 6).

Enumeration of adherent populations after $24 \mathrm{~h}$ (Table 1) indicated that biofilms of the two wild-type strains contained similar numbers of attached cells under steady-state conditions. These were 6-9 times greater than those of mutant 1001-92' (hypha ${ }^{-}$) and 17-26 times greater than those of strain 1001-FR (yeast ${ }^{-}$). Growth rates of the wild-type biofilms were also similar, and appeared to be 7-9 times greater than those of strain 1001-92' biofilms and c. 200 times greater than those of strain 1001-FR biofilms (Table 1).

Steady-state biofilms of all four strains were examined by SEM. This revealed, surprisingly, that both wildtype strains (GDH 2346 and 1001) produced biofilms containing only budding yeast cells on the cellulose surface (Fig. 7A and B). Indeed, the appearance of these biofilms resembled that of adherent populations formed by the mutant strain 1001-92' (Fig. 7C). All three strains generated relatively dense biofilms, although overall coverage of the filter surface was less extensive with the mutant strain. Biofilm formation by the hypha ${ }^{-}$mutant, strain 1001-FR, was confined to the uppermost section of the cellulose filter, close to the point of inoculation and medium influx. Sections of filter taken nearer the distal (medium efflux) end were devoid of hyphal growth. Evidently, hyphae were unable to colonise the body of the filter to any significant extent, with the result that a thick hyphal mat was formed on the top surface (Fig. 7D).

\section{Susceptibility of biofilm cells to amphotericin B}

Intact biofilms, resuspended biofilm cells and daughter cells of strain 1001 and its morphological mutants were exposed to amphotericin $\mathrm{B}$ at a concentration of $0.1 \mathrm{mg} / \mathrm{L}$ for $1 \mathrm{~h}$ at $37^{\circ} \mathrm{C}$. All biofilms were resistant to this treatment (Table 2). However, cells resuspended from any of the biofilms were $c$. $20 \%$ more susceptible than intact biofilm populations. Daughter cells from the parent strain and the hypha ${ }^{-}$mutant (strain 1001-FR) were even more susceptible to the drug. The yeast ${ }^{-}$ mutant (strain 1001-92'), on the other hand, produced daughter cells which were just as resistant as resuspended biofilm cells (Table 2).

\section{Discussion}

Structural organisation is a characteristic property of microbial biofilms and it is the feature which distinguishes them from conventional planktonic cultures. Studies with bacterial biofilms have established the bacterial microcolony as the basic structural and functional unit [7]. Microcolonies consist of aggregates of cells of a single, or several, species which are held together by a matrix of extracellular material. The advent of modern imaging techniques such as confocal scanning laser microscopy has allowed biofilms to be examined in a living, fully hydrated state. Such investigations have revealed that biofilms growing in aqueous environments frequently consist of mushroomshaped microcolonies separated by water-filled channels which facilitate transport through the biofilm [7]. Another characteristic biofilm property is a thin 'base film' which can range from a sparse monolayer of cells to a structure several layers thick [18]. Although the existence of these features is well documented for bacterial biofilms, there is little corresponding information on the structure of fungal biofilms and this is the first report of an investigation of the role of morphogenesis in biofilm formation by a dimorphic pathogenic fungus.

The results show that two mutant strains of $C$. albicans, incapable of yeast and hyphal growth, respectively, were nevertheless both able to produce 


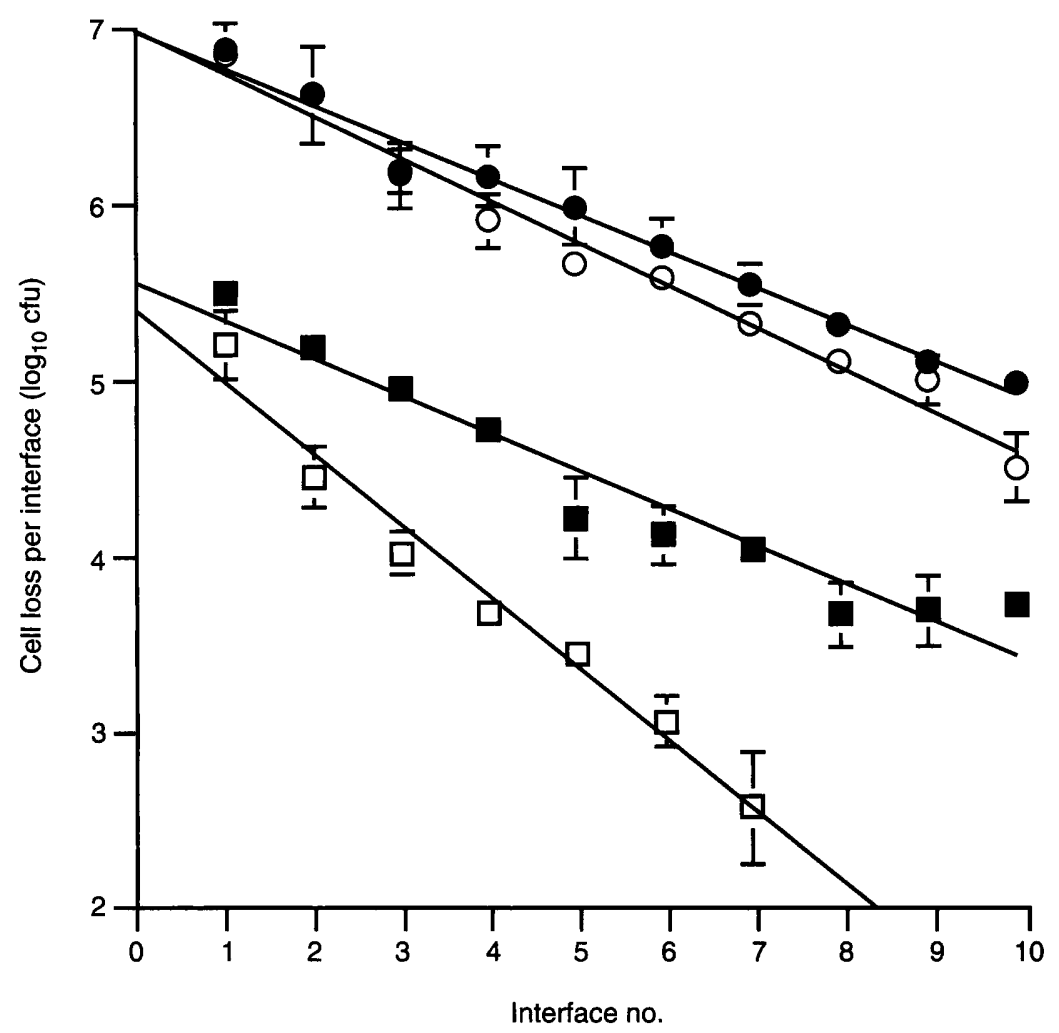

Fig. 5. Removal of $C$. albicans biofilm cells from PVC catheter disks by successive transfer through an air/liquid

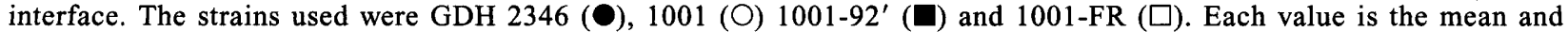
SEM of three independent experiments with viable counts done in duplicate.

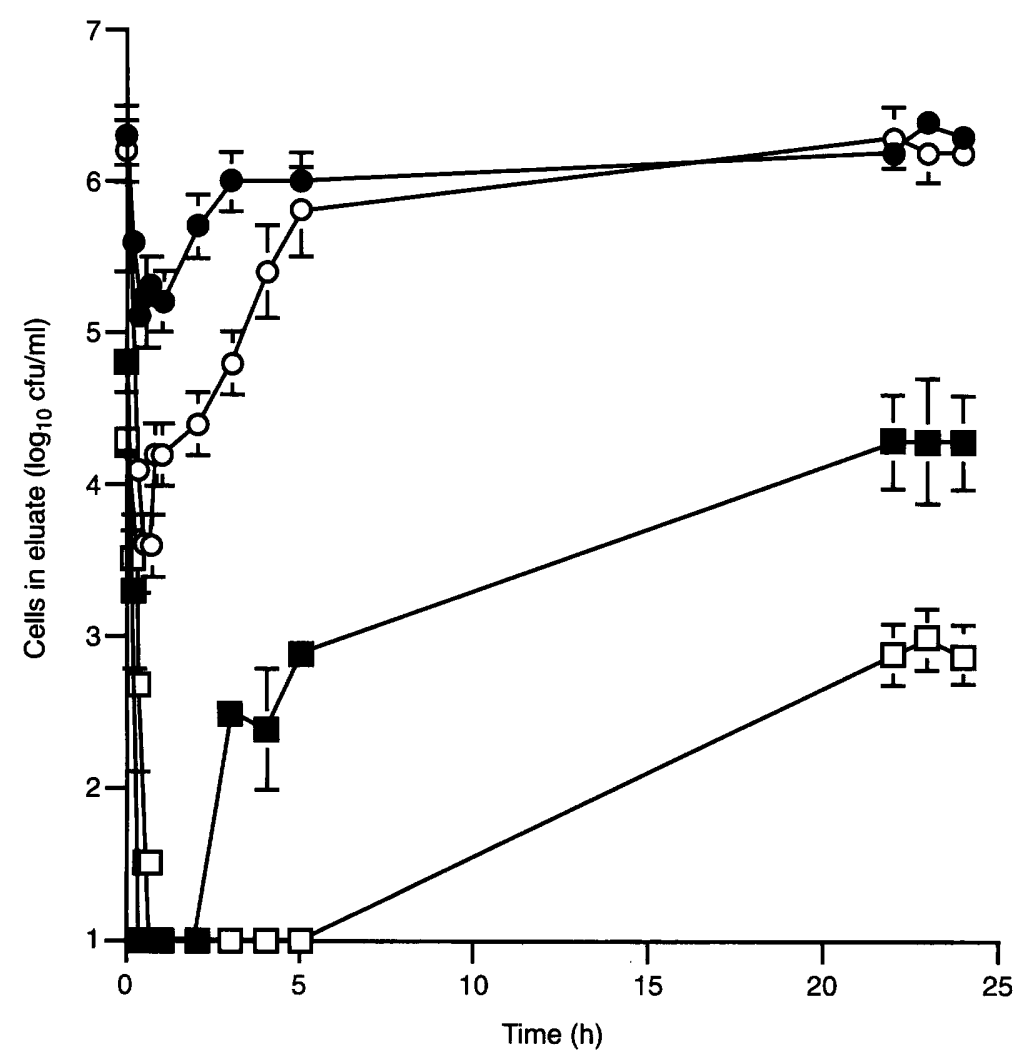

Fig. 6. Elution of $C$. albicans from cylindrical cellulose filters perfused with growth medium at a flow rate of $0.87 \mathrm{ml} / \mathrm{min}$. The strains used were GDH $2346(\bullet), 1001(\bigcirc), 1001-92^{\prime}(\square)$ and 1001-FR $(\square)$. Each value is the mean and SEM of three independent experiments with duplicate sampling. 
Table 1. Analysis of steady-state $C$. albicans biofilms grown for $24 \mathrm{~h}$ on cylindrical cellulose filters*

\begin{tabular}{lcc}
\hline $\begin{array}{l}\text { Strain } \\
\text { no. }\end{array}$ & $\begin{array}{c}\text { Mean (SEM) } \\
\text { adherent population } \\
\left(10^{8} \mathrm{cfu}\right)\end{array}$ & $\begin{array}{c}\text { Mean (SEM) } \\
\text { growth rate } \\
(/ \mathrm{h})\end{array}$ \\
\hline GDH 2346 & $34.1(11.8)$ & $0.043(0.012)$ \\
1001 & $22.0(3.1)$ & $0.033(0.008)$ \\
$1001-92^{\prime}$ & $3.8(1.6)$ & $0.005(0.002)$ \\
$1001-\mathrm{FR}$ & $1.3(0.1)$ & $0.0002(0.0001)$ \\
\hline
\end{tabular}

*Data are means and SEM of at least three independent experiments.

${ }^{\dagger}$ Data for strain GDH 2346 are taken from reference [14].
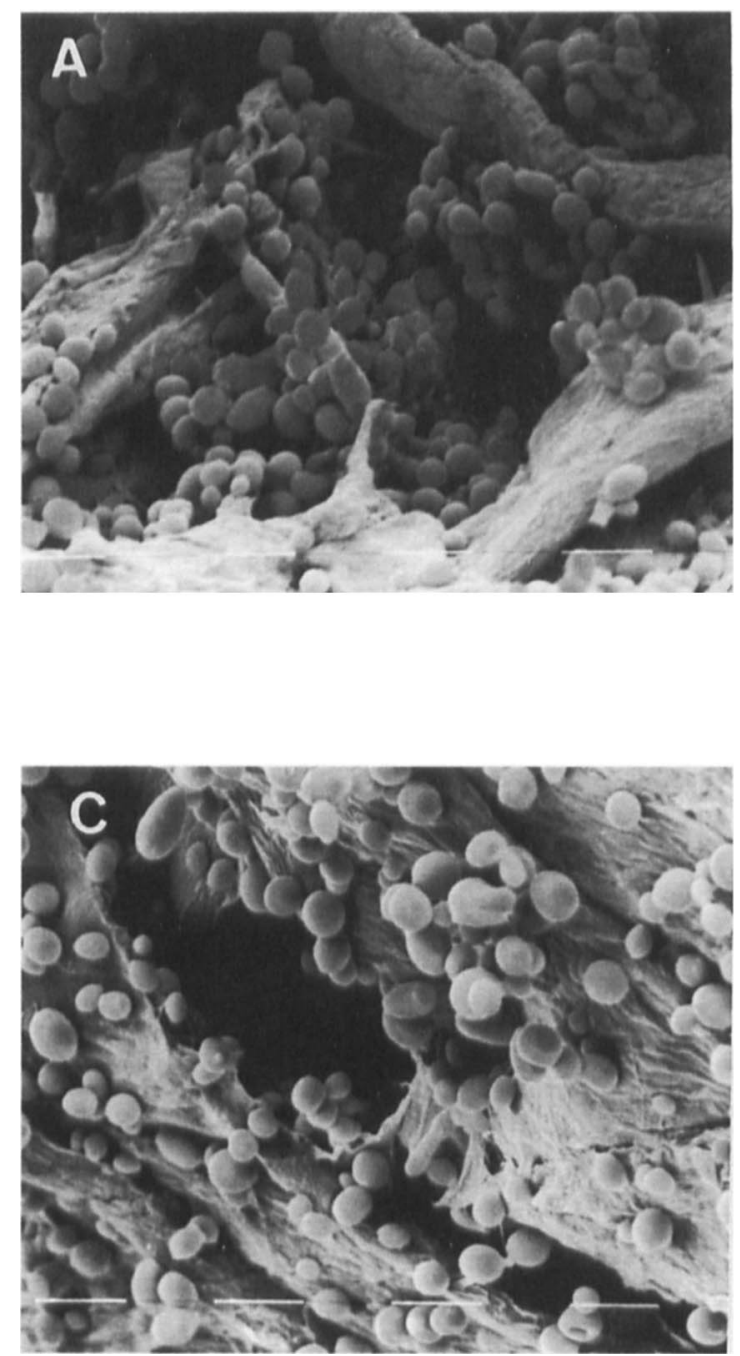

biofilms, indicating that dimorphism is not an absolute prerequisite for biofilm formation. However, the extent of biofilm formation on PVC disks differed between the two mutants. Strain 1001-92' (hypha ${ }^{-}$) produced biofilms that appeared to be less deep, as judged by SEM, than those of either strain 1001-FR (yeast ${ }^{-}$) or the wild type. These SEM observations are consistent with previous quantitative measurements of biofilm growth by the same strains, involving dry weight, colorimetric and radio-isotope assays. During a survey of $15 \mathrm{C}$. albicans isolates [10], it was noted that
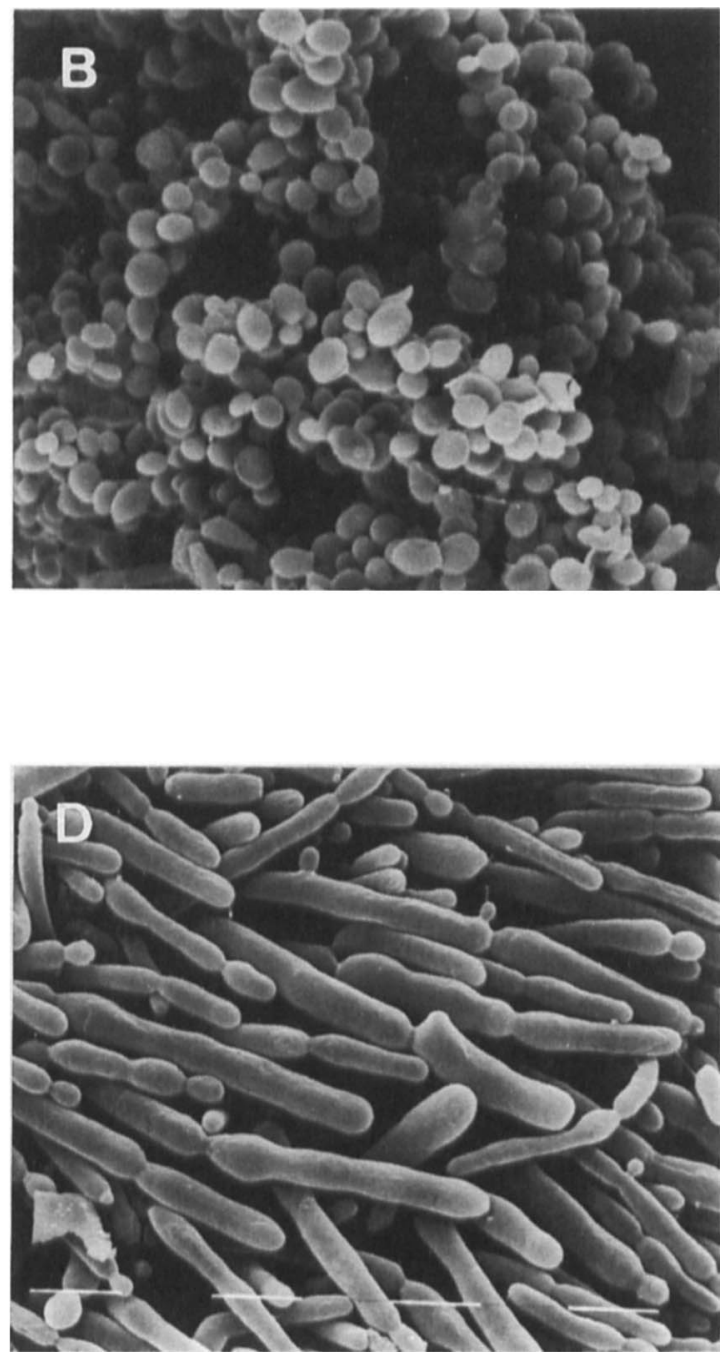

Fig. 7. SEM of biofilms formed by C. albicans strains GDH 2346 (A), 1001 (B), 1001-92' (C) and 1001-FR (D) after growth on cylindrical cellulose filters for $24 \mathrm{~h}$. Bar, $10 \mu \mathrm{m}$.

Table 2. Survival of biofilm cells of $C$. albicans after treatment with amphotericin $\mathrm{B}^{*}$

\begin{tabular}{lccc}
\hline & \multicolumn{3}{c}{ Mean (SEM) percentage survival of $C$. albicans cells } \\
\cline { 2 - 4 } Strain & resuspended biofilm & \\
no. & intact biofilms & cells & daughter cells \\
\hline 1001 & $100.5(11.7)$ & $86.6(5.4)$ & $59.6(12.2)$ \\
$1001-92^{\prime}$ & $100.8(21.9)$ & $78.8(7.4)$ & $82.1(7.8)$ \\
$1001-$ FR & $106.0(2.5)$ & $75.5(5.7)$ & $45.8(11.2)$ \\
\hline
\end{tabular}

${ }^{*}$ Data are means and SEM of three independent experiments. 
strains 1001 and 1001-FR gave similar values for all three parameters measured, whereas the values obtained with mutant $1001-92^{\prime}$ were significantly lower.

Biofilms of both wild-type strains on PVC disks appeared to consist of two distinct layers: a basal region consisting largely of densely packed yeast cells and an overlying thicker, but more open, hyphal layer. Although this bilayer structure was occasionally evident by SEM, it could be seen more clearly in thin sections of biofilms viewed by light microscopy (Fig. 2). The absence of one or other of the two layers was apparent in biofilms of the mutant strains. Strain $1001-92^{\prime}$ is able to form only a relatively thin biofilm consisting of the basal yeast layer. Presumably, this corresponds to the 'base film' which is well characterised in bacterial biofilms [18]. In contrast, strain 1001-FR forms a thicker, hyphal biofilm equivalent to the outer zone of the bilayer structure characteristic of wild-type strains. Interestingly, biofilms formed by this strain were much more easily detached from the PVC surface than those of the other strains (Fig. 5), suggesting that the basal yeast layer plays an important role in anchoring the biofilm to the surface. Although early studies indicated that germtubes of $C$. albicans were more adhesive than yeast cells [19], there is now some evidence that adhesion is inversely related to the proportion of hyphae in cultures containing a mixture of morphological forms, and that elongated hyphae can actually interfere with the initial stages of the attachment process [20].

Biofilms were formed by all four $C$. albicans strains on cylindrical cellulose filters, but the morphological mutants grew at much slower rates and took considerably longer to reach steady state than the wild-type strains. However, the mutants also displayed lower growth rates in batch culture. Both SEM and quantitative determinations of adherent cell populations indicated that the two wild-type strains colonised the filters more efficiently than the mutants although, surprisingly, neither wild-type strain produced hyphae in this system. Exclusively yeast-form biofilms have been reported previously with C. albicans GDH 2346 growing on cylindrical cellulose filters [14], but not on cellulose acetate membrane filters [13], suggesting that morphogenesis is dependent on highly specific contact-induced gene expression. Unexpectedly, biofilms produced by strain 1001-FR (yeast ${ }^{-}$) took the form of very thick, dense hyphal mats which were confined to the top section of the filter. The reason for the inability of this strain to colonise the rest of the filter is not clear. It may be related to a possible deficiency of oxygen inside the cylindrical filter although, as previously noted [14], such conditions usually favour hyphal growth.

Earlier reports from this laboratory have described the resistance of $C$. albicans biofilms to a range of antifungal agents $[11,13,14]$. The results of the present study show further that the morphological form of a biofilm does not affect its susceptibility to drugs. Intact biofilms of all four strains grown on cellulose filters were resistant to treatment with amphotericin B. Moreover, resuspended biofilm cells of all strains were c. $20 \%$ more susceptible to the drug than the corresponding intact biofilms. An increased susceptibility of resuspended cells to amphotericin B has been reported previously [14], and may be due to the loss of a protective effect afforded by the extracellular matrix. The presence of a fairly extensive matrix would be anticipated in a flow system of the type used here [12], although the fixation procedures used for SEM did not preserve this feature. Daughter cells eluted from $C$. albicans biofilms are characteristically more susceptible to amphotericin B than resuspended biofilm cells $[13,14]$. This result was obtained for all strains tested except the hypha ${ }^{-}$ mutant, 1001-92', whose daughter cells were more tolerant of the drug, a finding which remains unexplained.

The ability of $C$. albicans to exist in more than one morphological form has long been regarded as significant in pathogenesis, particularly in relation to the penetration of host tissues. This study has shown that morphogenesis also promotes the formation and persistence of adherent cell populations. C. albicans strains capable of dimorphism form biofilms on catheter disks consisting of a basal yeast layer, which serves as an anchor to the substratum, and an outer hyphal layer, which has a more open architecture likely to facilitate the transport of nutrients. Overall, the structure has several features in common with those of bacterial biofilms $[7,8,18]$. Recent investigations have demonstrated the involvement of cell-to-cell signalling in biofilm development by Pseudomonas aeruginosa [21]. A specific signalling mutant, deficient in the synthesis of acylhomoserine lactones, formed flat, thin biofilms which were devoid of microcolonies. These biofilms appear to bear a remarkable similarity to those of $C$. albicans 1001-92' which also consist of an undifferentiated base layer. However, whether an analogous cell-to-cell signalling system participates in the development of $C$. albicans biofilms remains to be determined.

This work was supported by grant $94 / 22$ A from the Sir Jules Thorn Charitable Trust. We are indebted to L. Tetley and M. Mullin for assistance with the microscopy.

\section{References}

1. Costerton JW, Cheng KJ, Geesey GG et al. Bacterial biofilms in nature and disease. Annu Rev Microbiol 1987; 41: 435-464.

2. Costerton, JW, Lewandowski Z, Caldwell DE, Korber DR, Lappin-Scott HM. Microbial biofilms. Annu Rev Microbiol 1995; 49: $711-745$.

3. Dougherty SH. Pathobiology of infection in prosthetic devices. Rev Infect Dis 1988; 10: 1102-1117.

4. Gristina AG. Biomaterial-centered infection: microbial ad- 
hesion versus tissue integration. Science 1987; 237: $1588-1595$.

5. Tunney MM, Gorman SP, Patrick S. Infection associated with medical devices. Rev Med Microbiol 1996; 7: 195-205.

6. Fridkin SK, Jarvis WR. Epidemiology of nosocomial fungal infections. Clin Microbiol Rev 1996; 9: 499-511.

7. Costerton JW. Overview of microbial biofilms. $J$ Ind Microbiol 1995; 15: 137-140.

8. Stoodley P, Boyle JD, Dodds I, Lappin-Scott HM. Consensus model of biofilm structure. In: Wimpenny J, Handley P, Gilbert P, Lappin-Scott H, Jones $M$ (eds) Biofilms: community interactions and control. Cardiff, Bioline. 1997: 1-9.

9. Brown MRW, Gilbert P. Sensitivity of biofilms to antimicrobial agents. J Appl Bacteriol 1993; 74 Suppl: 87S-97S.

10. Hawser SP, Douglas LJ. Biofilm formation by Candida species on the surface of catheter materials in vitro. Infect Immun 1994; 62: 915-921.

11. Hawser SP, Douglas LJ. Resistance of Candida albicans biofilms to antifungal agents in vitro. Antimicrob Agents Chemother 1995; 39: 2128-2131.

12. Hawser SP, Baillie GS, Douglas LJ. Production of extracellular matrix by Candida albicans biofilms. J Med Microbiol 1998; 47: $253-256$.

13. Baillie GS, Douglas LJ. Effect of growth rate on resistance of Candida albicans biofilms to antifungal agents. Antimicrob Agents Chemother 1998; 42: 1900-1905.
14. Baillie GS, Douglas LJ. Iron-limited biofilms of Candida albicans and their susceptibility to amphotericin B. Antimicrob Agents Chemother 1998; 42: 2146-2149.

15. Pomes R, Gil C, Nombela C. Genetic analysis of Candida albicans morphological mutants. J Gen Microbiol 1985; 131: 2107-2113.

16. Nombela C, Pomes R, Gil C. Protoplasts fusion hybrids from Candida albicans morphological mutants. Crit Rev Microbiol 1987; 15: 79-85.

17. Eginton PJ, Gibson H, Holah J, Handley PS, Gilbert P Quantification of the ease of removal of bacteria from surfaces. $J$ Ind Microbiol 1995; 15: 305-310.

18. Handley PS. Is there a universal biofilm structure? In: Wimpenny J, Handley P, Gilbert P, Lappin-Scott H (eds) The life and death of biofilm. Cardiff, Bioline. 1995: 21-25.

19. Kimura LH, Pearsall NN. Relationship between germination of Candida albicans and increased adherence to human buccal epithelial cells. Infect Immun 1980; 28: 464-468.

20. Vargas K, Wertz PW, Drake D, Morrow B, Soll DR. Differences in adhesion of Candida albicans 3153A cells exhibiting switch phenotypes to buccal epithelium and stratum corneum. Infect Immun 1994; 62: 1328-1335.

21. Davies DG, Parsek MR, Pearson JP, Iglewski BH, Costerton JW, Greenberg EP. The involvement of cell-to-cell signals in the development of a bacterial biofilm. Science 1998; 280: 295-298. 